\title{
Excitation of transverse magnetic tube waves in stellar convection zones
}

\section{Effects of metallicity on wave energy spectra and fluxes}

\author{
Z. E. Musielak ${ }^{1,2}$ and P. Ulmschneider ${ }^{2}$ \\ 1 Department of Physics, University of Texas at Arlington, Arlington, TX 76019, USA \\ 2 Institut für Theoretische Astrophysik der Universität Heidelberg, Tiergartenstr. 15, 69121 Heidelberg, Germany
}

Received 24 July 2001 / Accepted 3 December 2001

\begin{abstract}
In the previous two papers of this series, we developed an analytical method describing the generation of transverse tube waves in stellar convection zones and used it to compute the wave energy spectra and fluxes for late-type stars with the solar metal abundance (population I stars). We now extend these calculations to population II stars with effective temperatures ranging from $T_{\text {eff }}=2500 \mathrm{~K}$ to $10000 \mathrm{~K}$, gravities $\log g=3-5$, and with three different metal abundances: $1 / 10,1 / 100$ and $1 / 1000$ of solar metallicity. The obtained results are valid only for a single magnetic flux tube and they show that the effects of metallicity are important only for cool stars with $T_{\text {eff }}<6000 \mathrm{~K}$ and that the amount of the generated wave energy decreases roughly by an order of magnitude for every decrease of the metallicity by an order of magnitude. The maximum wave energy flux generated in population II stars is $3 \times 10^{9} \mathrm{erg} \mathrm{cm}^{-2} \mathrm{~s}^{-1}$ and it is practically the same for stars of different gravities and metallicities.
\end{abstract}

Key words. methods: analytical - stars: chromosphere - stars: coronae - stars: magnetic fields - MHD - waves

\section{Introduction}

In our previous work (Musielak \& Ulmschneider 2001, hereafter called Paper I), we developed a general analytical treatment of the interaction between a single magnetic flux tube and the external turbulent motions, and derived the basic formulae for the efficiency of generation of linear transverse tube waves in stellar convection zones. In Paper II (Musielak \& Ulmschneider 2002), these formulae were used to compute the wave energy spectra and fluxes for late-type stars with solar metal abundance (population I stars). We now study the effects caused by different metallicity on the generated transverse wave energy spectra and fluxes. Obviously, the stellar populations differ in their content of metals, which refer to all chemical elements except hydrogen and helium.

Population I stars like the Sun have a high metal abundance, while population II stars have $1 / 10$ to $1 / 100$, or sometimes even $1 / 1000$ of the solar metal abundance. We consider late-type stars of population II with three different metal abundances using opacity tables calculated with the ATLAS-program by Kurucz $(1992,1996)$. For comparison, we also compute transverse wave energy spectra and

Send offprint requests to: P. Ulmschneider, e-mail: ulm@ita.uni-heidelberg.de fluxes by using Kurucz opacity tables for solar metal abundances (see Ulmschneider et al. 1999), and opacity tables originally described by Bohn (1984) which were used by us in Papers I and II.

The results presented in this paper were obtained for stars with effective temperatures ranging from $T_{\text {eff }}=$ $2500 \mathrm{~K}$ to $10000 \mathrm{~K}$ and gravities in the range of $\log g=$ $3-5$. The computed spectra and fluxes can be used to construct theoretical chromosphere models for these stars (e.g., Cuntz et al. 1999; Fawzy 2001; Ulmschneider et al. 2001a). A brief description of the computational method and the opacity tables used in this paper is given in Sects. 2 and 3, respectively. The generated stellar wave energy spectra and fluxes of transverse tube waves are presented and discussed in Sect. 4. Section 5 gives our conclusions.

\section{Method of computation}

The basic formulae describing the generation of transverse tube waves in stellar convection zones are given in Paper II (see Eqs. (7) through (13)), and they will not be repeated here. These formulae are valid for a single and vertically oriented magnetic flux tube that is thin and circular, and embedded in an atmosphere of a star of given $T_{\text {eff }}$ and $\log g$. In addition, it is assumed that 
there are no longitudinal flows inside the tube. To perform our calculation of stellar wave energy spectra and fluxes, we must specify the field strength inside the magnetic flux tube. Since the strength of this field cannot currently be determined by stellar observations (Saar 1996; Rüedi et al. 1997), we guide ourselves by solar observations which show that the typical field strength in solar magnetic flux tubes at the solar surface is $B_{\mathrm{o}}=1500 \mathrm{G}$ (e.g., Solanki 1993); this corresponds to about $B_{\mathrm{o}}=0.85 B_{\text {eq }}$, where $B_{\text {eq }}=\sqrt{8 \pi p_{\mathrm{e}}}$ is the equipartition field strength and $p_{\mathrm{e}}$ the external gas pressure at the solar surface. In Paper II, we considered three different values magnetic field strength, $B_{\mathrm{o}} / B_{\text {eq }}=0.75,0.85$ and 0.95 , for population I stars. In the present paper we take the same values for population II stars. Note that the field strength $B_{\mathrm{o}}$ is specified at the atmospheric optical depth $\tau_{5000}=1$.

The structure of the non-magnetized medium that surrounds the tube is calculated by using a stellar envelope computer code described by Bohn (1984) and later modified by Theurer (1993) and Ulmschneider et al. (1996). The input parameters to the code are: $T_{\text {eff }}, g$, and the mixing-length parameter $\alpha=l_{\text {mix }} / H$, where $l_{\text {mix }}$ is the mixing-length and $H$ is the pressure scale height. A value of $\alpha=2$ (see Paper II) is assumed in most of our calculations, however, we have also computed wave energy fluxes with $\alpha=1$ and 1.5 for stars with $\log g=4$. For a given value of $\alpha$, the code gives a stellar convection zone model, which is then employed as model for the external medium. On basis of this model, the horizontal pressure balance and the conservation of magnetic flux, we compute the structure of the background medium inside the tube by specifying the value of $B_{\mathrm{o}}$. The tube model is computed by assuming the so-called thin flux tube approximation, which requires the tube diameter at the stellar surface to be roughly equal to the local scale height (see Paper I). To calculate these models, we must have opacity tables valid for the reduced metallicity observed in population II stars. In the following, we describe our choice of the opacity tables for these stars.

\section{Opacity tables}

The results presented in Paper II for population I stars were obtained by using opacity tables compiled by Bohn (1984) and Theurer (1993) from various opacity tables given by Alexander (1975, 1989), Cox \& Tabor (1976), Yorke (1979, 1980), Meyer-Hofmeister (1982), and Weiss (1990). Here, we refer to these tables as Bohn opacity and they are valid for the solar chemical abundance, i.e. abundance by mass of hydrogen $X_{\mathrm{m}}=0.70$, helium $Y_{\mathrm{m}}=0.28$ and of metals $Z_{\mathrm{m}}=2 \times 10^{-2}$. To account for the reduced metallicity observed in population II stars, we use the opacity tables calculated with the ATLAS-program of Kurucz $(1992,1996)$. We refer to these tables as the Kurucz opacity and they are available for the following solar and non-solar metal abundances: $Z_{\mathrm{m}}=2 \times 10^{-2} \widehat{=}[0]$, $Z_{\mathrm{m}}=2 \times 10^{-3} \widehat{=}[-1], Z_{\mathrm{m}}=2 \times 10^{-4} \widehat{=}[-2]$, and $Z_{\mathrm{m}}=2 \times 10^{-5} \widehat{=}[-3]$, where the logarithm of the reduction factor is given in square brackets - this notation for metallicity will be used in the remaining part of this paper.

Thus, the wave energy spectra and fluxes presented in this paper for population II stars are obtained with the Kurucz opacity $[-1],[-2]$ and $[-3]$. For comparison, we also compute the spectra and fluxes by using the Kurucz opacity [0] and the Bohn opacity. The range of validity of these opacity tables is shown and discussed by Ulmschneider et al. (1999 - see their Fig. 1). The limited range of these opacity tables imposes some restrictions on the convection zone models that can be computed. For example, high temperatures and densities present in deep layers of stellar convection zones typically exceed the range of these parameters in the opacity tables.

Fortunately, the most efficient wave generation takes place mainly in the subphotospheric layers and the contribution from the deep layers is always negligible. Hence, the deepest layer in our convection zone models is calculated with the last entry of the opacity table before the range of this table is exceeded. Another problem is the low temperature and density limit of the opacity tables and uncertainties in the values of opacities for low $T_{\text {eff }}$. Because of these problems, we consider only stars with $T_{\text {eff }} \geq 2500 \mathrm{~K}$ and terminate our computations of stellar convection zone models if temperatures and densities in the outermost layers of these models fall outside the lower boundary of the opacity table.

\section{Results and discussion}

Since the method developed in Paper I is valid for propagating transverse tube waves, we assume that the wave frequency domain extends from $\omega=1.002 \Omega_{k}$ to $57 \Omega_{k}$, where $\Omega_{k}=c_{k} / 4 H$ is the cutoff frequency for these waves, $c_{k}$ is the characteristic wave velocity, and $H$ is the scale height (see Paper I or II). The wave energy fluxes are computed by performing the Laguerre integration over the wave frequency $\omega$ by using 32 points within the frequency domain. All presented results are obtained for a single magnetic flux tube embedded in the convection zone of a late-type star of different $T_{\text {eff }}$ and $\log g$. This means that the filling factor, representing the number of magnetic flux tubes per unit area of the stellar surface, will not be discussed here.

\subsection{Wave energy spectra}

Typical stellar wave energy spectra calculated for stars of different metallicity but with the same effective temperature, $T_{\text {eff }}=4000 \mathrm{~K}$, and gravity, $\log g=4$, are shown in Fig. 1. It is seen that the shapes of the wave energy spectra for population II stars and metallicities $[0],[-1]$, $[-2]$ and $[-3]$ are quite similar but noticeably decrease in magnitude with decreasing metallicity, particularly at high frequency. This effect can be explained as follows.

The top boundary of the convection zone is reached and convection stops when the rising convective gas 


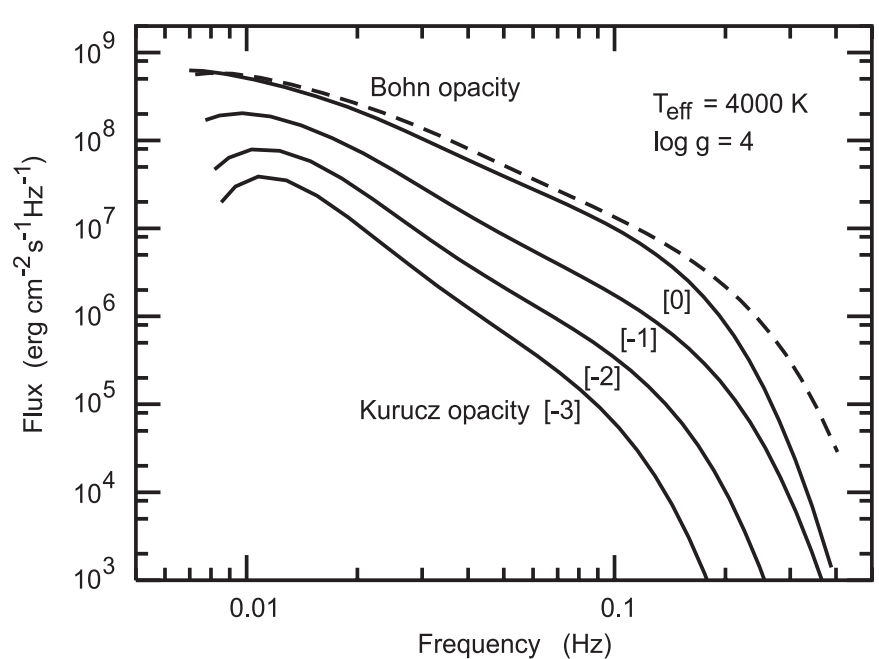

Fig. 1. Transverse wave energy spectra plotted for stars with $T_{\text {eff }}=4000 \mathrm{~K}$ and $\log g=4$, and different metallicities. The results were obtained for a mixing-length parameter $\alpha=2$ and a magnetic field strength $B_{\mathrm{o}}=0.85 B_{\text {eq }}$ at $\tau_{5000}=1$.

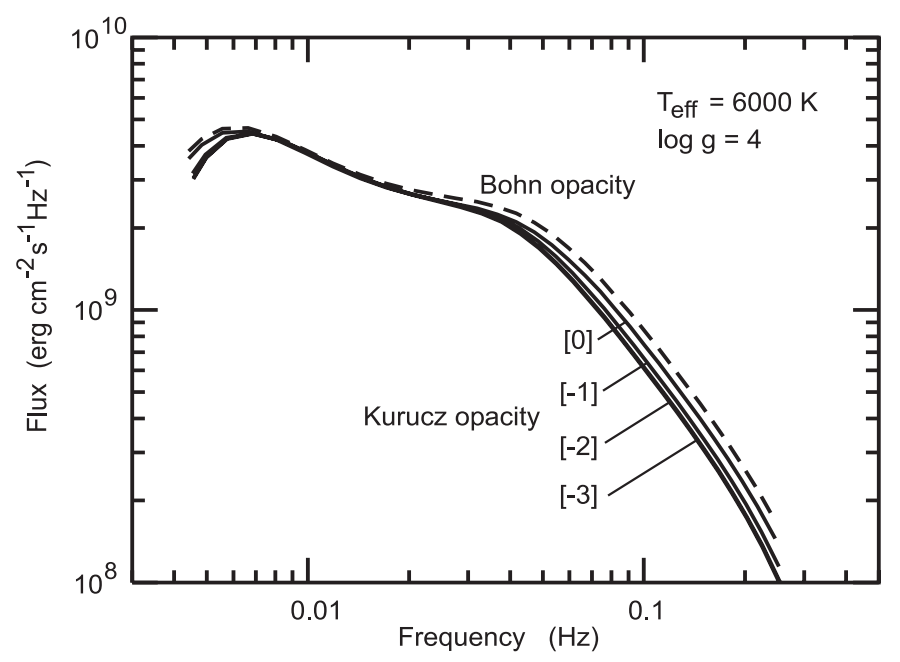

Fig. 2. Same as Fig. 1 but for $T_{\text {eff }}=6000 \mathrm{~K}$.

bubbles near the stellar surface come to a height, where the opacity is so low that the excess thermal energy of the bubbles can be radiated directly into space. As the stellar convection zone carries the total stellar radiative flux $\sigma T_{\text {eff }}^{4}$, a lowering of the opacity has the consequence that this height is reached at deeper layers where the density $\rho$ is higher and because one has $\rho v_{\text {conv }}^{3} \approx \sigma T_{\text {eff }}^{4}$, the convective velocity $v_{\text {conv }}$ is correspondingly smaller. Since the wave generation depends on a high power of the convective velocity, which has its maximum near the top of the convection zone, it is strongly affected by this lowering.

On the other hand the wave energy spectra for stars with $T_{\text {eff }}=6000 \mathrm{~K}$ and $\log g=4$ (shown in Fig. 2) are almost identical and show very little dependence on metallicity. This is because in hot stars the opacity is mainly due to hydrogen, which is not affected by changing the metallicity, while in cool stars the opacity is mainly due to metals and reacts strongly to the lowering of the metal

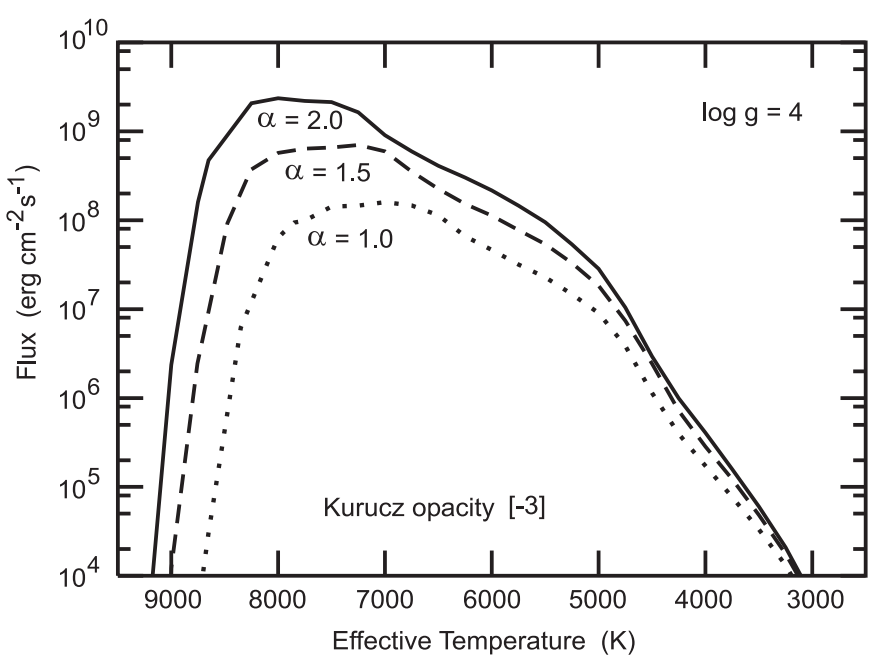

Fig. 3. Transverse wave energy fluxes computed for stars of different spectral types are plotted for three different values of the mixing-length parameter $\alpha=1,1.5$ and 2. The fluxes were obtained for stars with $\log g=4$, metallicity [-3], and a magnetic field strength $B_{\mathrm{o}}=0.85 B_{\text {eq }}$ at $\tau_{5000}=1$.

abundance. Note that the presented spectra were computed by taking $\alpha=2$ and $B_{\mathrm{o}} / B_{\text {eq }}=0.85$.

By comparing the spectra plotted in Figs. 1 and 2, we draw the following general conclusions. First, the effects of metallicity are prominent in cool stars but minor in hot stars. Second, the amount of wave energy carried by transverse tube waves of the same frequency in cool stars strongly decreases with decreasing metallicity. Third, the generated wave energy flux for a given frequency is always much higher for hot stars than for cool stars - this is simply caused by higher convective velocities in the former. Fourth, the computed wave energy spectra are relatively flat at high $T_{\text {eff }}$, yet become much steeper when $T_{\text {eff }}$ is decreased. Finally, for both hot and cool stars the transverse wave energy spectra extend more than one order of magnitude above the cut-off frequency.

\subsection{Dependence on physical parameters}

There are two basic physical parameters in our calculations. One is the mixing-length parameter $\alpha$ and the other the strength of the magnetic field, $B_{\mathrm{o}} / B_{\text {eq }}$. To investigate the effects of these parameters on the computed stellar wave energy fluxes, we consider population II stars with $\log g=4$ and metallicity $[-3]$, and take $\alpha=1$, 1.5 and 2 for a fixed $B_{\mathrm{o}}=0.85 B_{\text {eq }}$, and alternatively $B_{\mathrm{o}} / B_{\text {eq }}=0.75,0.85$ and 0.95 for a fixed $\alpha=2$.

The dependence of the calculated fluxes on the parameters $\alpha$ and $B_{\mathrm{o}} / B_{\text {eq }}$ for stars of different $T_{\text {eff }}$ is shown in Figs. 3 and 4, respectively. According to Fig. 3, the $\alpha$-dependence is relatively weak for cool stars, however, significant differences in the generated fluxes are seen for hot stars. These differences can be explained by higher efficiency of stellar convection in hot stars and its high sensitivity to the value of $\alpha$. The dependence of the wave 


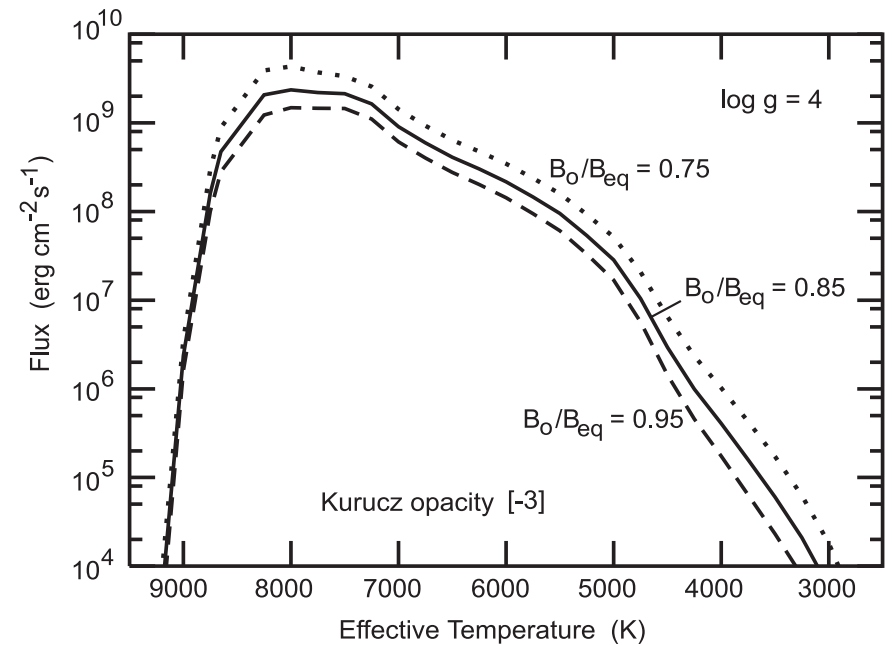

Fig. 4. Transverse wave energy fluxes computed for stars of different spectral types are plotted for three different values of the tube magnetic field: $B_{\mathrm{o}} / B_{\mathrm{eq}}=0.75,0.85$ and 0.95 specified at $\tau_{5000}=1$. The fluxes were obtained for stars with $\log g=4$, metallicity [-3], and a mixing-length parameter $\alpha=2$.

Table 1. Transverse wave energy fluxes, $F_{\mathrm{T}}\left(\mathrm{erg} \mathrm{cm}^{-2} \mathrm{~s}^{-1}\right)$ for different values of the mixing-length parameter $\alpha$ and tube magnetic field $B_{\mathrm{o}} / B_{\text {eq }}$. The fluxes were computed for a star with $T_{\text {eff }}=6000 \mathrm{~K}, \log g=4$ and metallicity [-3].

\begin{tabular}{|c||c|c|}
\hline$\alpha$ & $B_{\mathrm{o}} / B_{\text {eq }}$ & $F_{\mathrm{T}}$ \\
\hline \hline 1.0 & 0.85 & $4.6 \times 10^{7}$ \\
1.5 & 0.85 & $1.1 \times 10^{8}$ \\
2.0 & 0.85 & $2.2 \times 10^{8}$ \\
2.0 & 0.75 & $3.5 \times 10^{8}$ \\
2.0 & 0.95 & $1.4 \times 10^{8}$ \\
\hline
\end{tabular}

energy fluxes on $\alpha$ increases with increasing $T_{\text {eff }}$ and is especially prominent for stars with $T_{\text {eff }}>7000 \mathrm{~K}$.

On the other hand, the results of Fig. 4 show that the wave energy flux increases with decreasing magnetic field strength and stars of different $T_{\text {eff }}$ are equally affected. This dependence can be explained by the decreasing stiffness of the tube for weaker tube magnetic fields. Note that the dependence of the generated wave energy fluxes on both $\alpha$ and $B_{\mathrm{O}}$ found here for population II stars is very similar to that obtained in Paper II for population I stars.

The dependence of the wave energy fluxes on the mixing-length parameter $\alpha$ for a given star with $T_{\text {eff }}=$ $6000 \mathrm{~K}, \log g=4$ and metallicity [-3] is given in Table 1. Based on these results, we find that the dependence of $F_{\mathrm{T}}$ on $\alpha$ can be approximated by

$F_{\mathrm{T}} \approx 4.6 \times 10^{7} \alpha^{2.2} \mathrm{erg} \mathrm{cm}^{-2} \mathrm{~s}^{-1}$.

The derived $\alpha$-dependence is similar to that found for the transverse wave generation rate in the Sun (Paper I) and other population I stars (Paper II).

Table 1 also shows that the total wave energy flux $F_{\mathrm{T}}$ decreases with increasing magnetic field strength $B_{\mathrm{o}} / B_{\text {eq }}$.

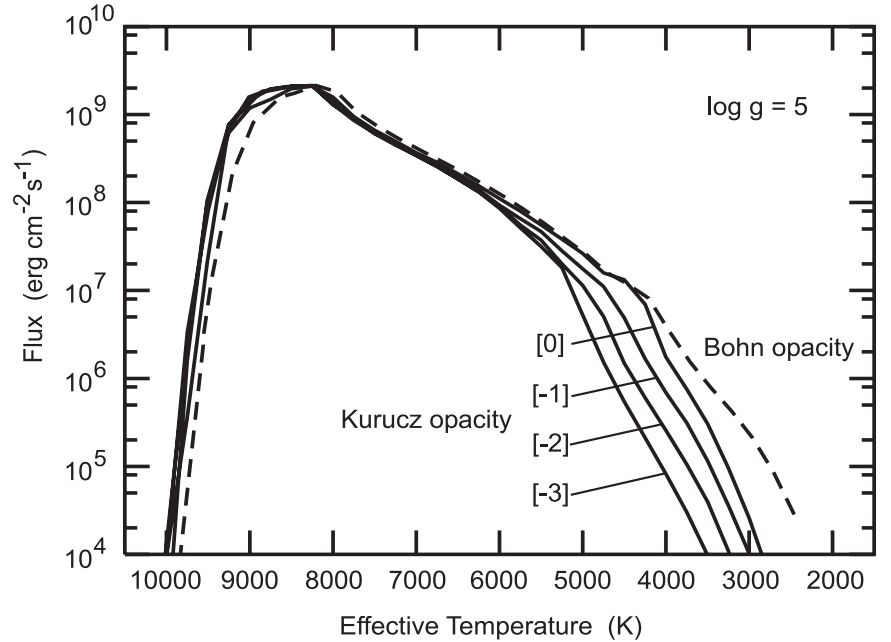

Fig. 5. Transverse wave energy fluxes computed for stars of different spectral types and different metallicities. The fluxes were obtained for stars with $\log g=5$, the mixing-length parameter $\alpha=2$, and for the tube magnetic field $B_{\mathrm{o}}=0.85 B_{\text {eq }}$ at $\tau_{5000}=1$.

This dependence can approximately be fitted by the following expression:

$F_{\mathrm{T}} \approx 1.2 \times 10^{8}\left(\frac{B_{\mathrm{o}}}{B_{\mathrm{eq}}}\right)^{-3.6} \mathrm{erg} \mathrm{cm}^{-2} \mathrm{~s}^{-1}$.

Again this dependence is very similar to that found for the Sun in Paper I and for population I stars in Paper II; this simply means that the dependence of the transverse wave generation on the tube magnetic field does not change when stellar metallicity is decreased.

Based on these results, we conclude that the effects caused by the mixing-length parameter, $\alpha$, and the tube magnetic field, $B_{\mathrm{o}} / B_{\text {eq }}$, on the rate of the generation of transverse tube waves are independent of stellar metallicity.

\subsection{Stellar wave energy fluxes}

The transverse wave energy fluxes computed for stars with different effective temperatures and metallicities are shown in Figs. 5, 6 and 7, which are arranged in order of decreasing gravity from $\log g=5$ to 3 . These figures clearly show that the effects of metallicity are important only in stars with $\log g=5$ and $T_{\text {eff }}<6000 \mathrm{~K}$, with $\log g=4$ and $T_{\text {eff }}<5500 \mathrm{~K}$, and with $\log g=3$ and $T_{\text {eff }}<5000 \mathrm{~K}$. It is also seen that the wave energy fluxes for cool population II stars decrease roughly by an order of magnitude for every decrease of the metallicity by an order of magnitude, and that this result is independent of stellar gravity.

Note that for cool stars there are also discrepancies between the wave energy fluxes calculated by using the Kurucz opacity tables with metallicity [0] and the Bohn opacity tables. As already explained by Ulmschneider et al. (1999), the most likely reason for these discrepancies is the limited range of these tables. 


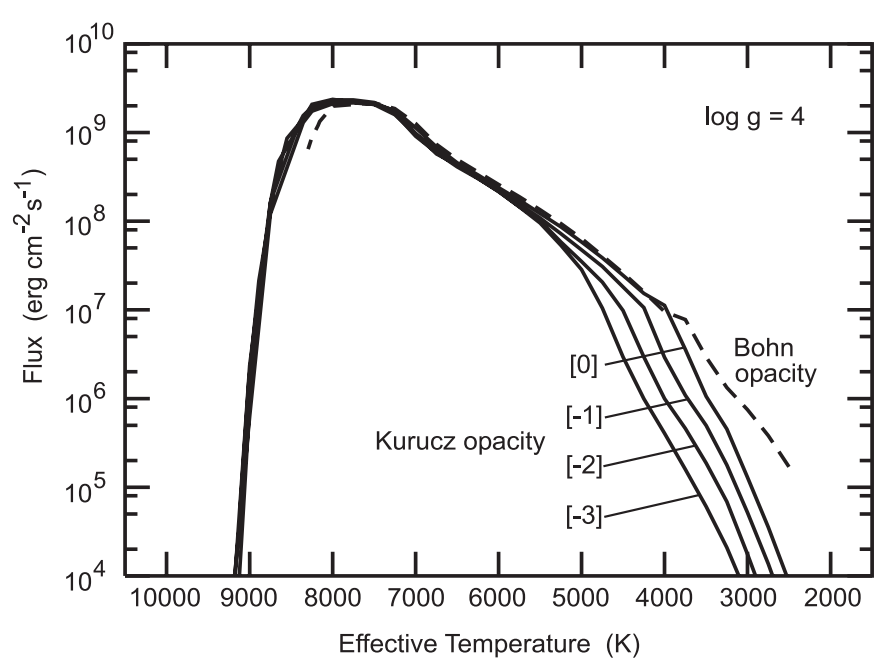

Fig. 6. Same as Fig. 5 but for $\log g=4$.

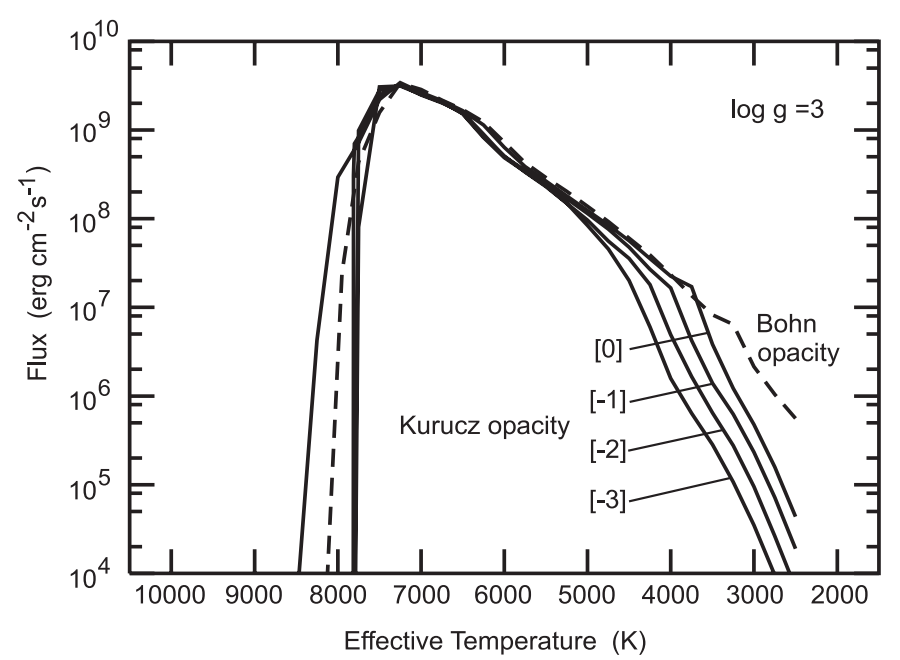

Fig. 7. Same as Fig. 5 but for $\log g=3$.

For hot stars with $\log g=5$ and 4 , the wave energy fluxes are practically identical (see Figs. 5 and 6, also Table 2). The stars with the highest temperatures, where the wave fluxes rise very rapidly, are strongly affected by the onset of convection. This is because of the onset of the hydrogen ionization which influences the adiabatic gradient and occurs at progressively lower $T_{\text {eff }}$ for lower gravity. The convection zones in these stars are very narrow and the convective velocities become very sensitive to the numerical integration steps. Hence, the resulting wave energy fluxes strongly vary with small changes in $T_{\text {eff }}$. This effect is especially important for giants with $\log g=3$ (see Fig. 7). In some of these stars, the maximum convective velocities become comparable to (or even higher than) the local sound speed; in this case, our method gives unrealistic results (see Papers I and II).

For cooler stars the convective velocities decrease and with it the transverse wave energy fluxes. The strong influence of the metallicity on these fluxes has already been discussed (see Sect. 4.1) and explained by the fact that for these stars the main contributers to the opacity is from

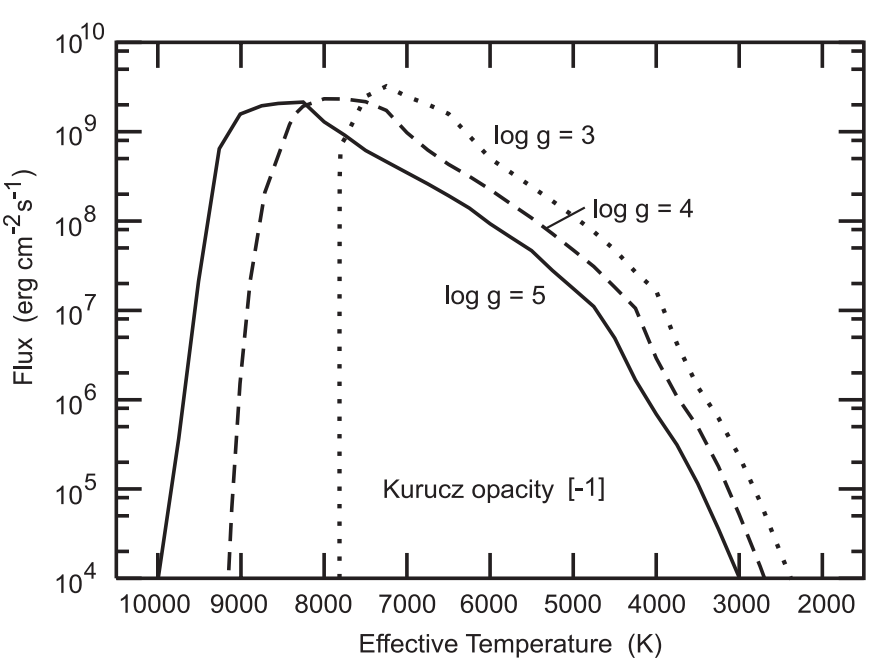

Fig. 8. Transverse wave energy fluxes computed for stars of different spectral types and different gravities. The fluxes were obtained for stars with the metallicity $[-1]$, the mixing-length parameter $\alpha=2$, and for the tube magnetic field $B_{\mathrm{o}}=0.85 B_{\text {eq }}$ at $\tau_{5000}=1$.

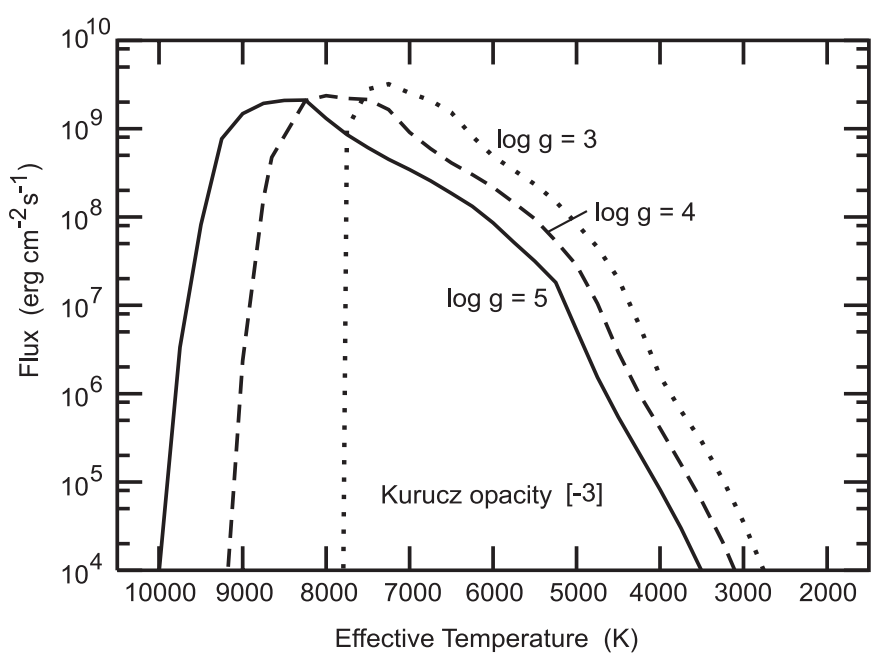

Fig. 9. Same as Fig. 8 but for the metallicity $[-3]$.

metals while for hot stars the opacity is mainly from hydrogen. The obtained results clearly indicate that metallicity does not affect hot stars but it is important for cool stars (see Figs. 5, 6 and 7; also Table 2).

The influence of gravity on the wave fluxes computed for stars of different metallicities $[-1]$ and $[-3]$ are shown in Figs. 8 and 9, respectively. The results were obtained for $\alpha=2$ and $B_{\mathrm{o}}=0.85 B_{\text {eq. }}$. Both figures show that the onset of convection at the hot side strongly restricts the range of $T_{\text {eff }}$ for which the efficient excitation of transverse tube waves occurs. It is largest for dwarfs with $\log g=5$ and the smallest for giants with $\log g=3$. Additional problem here is that for some of these giants the maximum convective velocities become large in an progressively narrower region and, as a result, the ratio of the maximum convective velocity to the local sound speed (the so-called convective Mach number) can exceed 1 (see Ulmschneider et al. 2001b, and their Fig. 1). We discussed this issue in 
Table 2. Wave energy fluxes, $F_{\mathrm{T}}\left(\mathrm{erg} \mathrm{cm}^{-2} \mathrm{~s}^{-1}\right)$, for upward propagating transverse tube waves in stars with different effective temperatures $T_{\text {eff }}$, gravity $\log g$, and metallicity. The fluxes were computed by using the mixing-length parameter $\alpha=2$ and the tube magnetic field $B_{\mathrm{o}}=0.85 B_{\text {eq }}$ at $\tau_{5000}=1$.

\begin{tabular}{|c||c|c|c|}
\hline$T_{\text {eff }}$ & $\log g=3$ & 4 & 5 \\
\hline \hline & & Metallicity [0] & \\
\hline 3000 & $4.7 \times 10^{5}$ & $1.3 \times 10^{5}$ & $2.6 \times 10^{4}$ \\
5000 & $1.2 \times 10^{8}$ & $5.8 \times 10^{7}$ & $2.6 \times 10^{7}$ \\
7000 & $2.7 \times 10^{9}$ & $1.1 \times 10^{9}$ & $3.7 \times 10^{8}$ \\
9000 & & $6.4 \times 10^{5}$ & $1.2 \times 10^{9}$ \\
\hline & & Metallicity [-1] & \\
\hline 3000 & $2.3 \times 10^{5}$ & $5.2 \times 10^{4}$ & $1.0 \times 10^{4}$ \\
5000 & $1.1 \times 10^{8}$ & $4.7 \times 10^{7}$ & $1.8 \times 10^{7}$ \\
7000 & $2.5 \times 10^{9}$ & $9.8 \times 10^{8}$ & $3.5 \times 10^{8}$ \\
9000 & & $1.5 \times 10^{6}$ & $1.6 \times 10^{9}$ \\
\hline & & Metallicity [-2] & \\
\hline 3000 & $9.5 \times 10^{4}$ & $1.8 \times 10^{4}$ & $2.3 \times 10^{3}$ \\
5000 & $9.6 \times 10^{7}$ & $3.5 \times 10^{7}$ & $1.1 \times 10^{7}$ \\
7000 & $2.5 \times 10^{9}$ & $9.6 \times 10^{8}$ & $3.4 \times 10^{8}$ \\
9000 & & $8.8 \times 10^{5}$ & $1.5 \times 10^{9}$ \\
\hline & & Metallicity [-3] & \\
\hline 3000 & $3.5 \times 10^{4}$ & $5.8 \times 10^{3}$ & $3.6 \times 10^{2}$ \\
5000 & $8.4 \times 10^{7}$ & $2.8 \times 10^{7}$ & $5.2 \times 10^{6}$ \\
7000 & $2.5 \times 10^{9}$ & $9.1 \times 10^{8}$ & $3.4 \times 10^{8}$ \\
9000 & & $2.4 \times 10^{6}$ & $1.5 \times 10^{9}$ \\
\hline
\end{tabular}

Paper II and concluded that the wave energy fluxes for $T_{\text {eff }}>8000 \mathrm{~K}$ and $\log g=4$, and for $T_{\text {eff }}>7000 \mathrm{~K}$ and $\log g=3$ might not be realistic. The same restrictions apply to the population II stars considered in the present paper.

Figures 8 and 9 also show that stars with lower gravities but similar effective temperature produce higher transverse wave energy fluxes. It is also seen that these fluxes rapidly increase with increasing $T_{\text {eff }}$ until they reach their maxima, which seem to be similar for stars of different gravities and metallicities. The maximum wave energy flux generated in population II stars is about $3 \times 10^{9} \mathrm{erg} \mathrm{cm}^{-2} \mathrm{~s}^{-1}$ and is practically the same for stars of different gravities and metallicities. This maximum flux occurs for A or early F-stars just before stellar convection ceases to exist at higher effective temperatures. By comparing the fluxes obtained with metallicity $[-1]$ and $[-3]$, one sees that the range of $T_{\text {eff }}$ for which the wave energy flux is higher than $1.0 \times 10^{4} \mathrm{erg} \mathrm{cm}^{-2} \mathrm{~s}^{-1}$ gets smaller with decreasing metallicity. This effect is most prominent for the coolest stars considered here (see Table 2).

As so far no other investigation of the effect of metallicity on the transverse tube wave generation is available, we now compare our results with those obtained by Ulmschneider et al. (1999) for the acoustic wave generation and by Musielak et al. (2001) for the longitudinal tube wave generation. These authors have shown that late-type stars can be divided into three groups labeled I, II and III, which represent regions in the HR diagram with different dependences of the acoustic and longitudinal tube wave generation on the stellar metal abundance. For hot stars of group I, there is no dependence of the generated wave energy fluxes on metallicity. For cool stars of group III, the wave generation strongly depends on the metallicity and the generated fluxes are lowered roughly by an order of magnitude for every decrease of the metallicity by an order of magnitude. However, stars of group II represent the transition between the other two groups and the generated acoustic and longitudinal wave energy fluxes become a sensitive function of the effective temperature.

The above conclusions are in full agreement with the results obtained in the present paper for transverse tube waves. This means that the effects of metallicity on the wave generation process are very similar for non-magnetic and magnetic waves. It must be also mentioned that increasing wave power with frequency at low frequencies seen in the transverse wave energy spectra (see Figs. 1 and 2) is very similar to that found for the acoustic and longitudinal wave energy spectra. Finally, the obtained results show that the efficiency of the generation of transverse tube waves in stellar convection zones is higher than that of acoustic and longitudinal tube waves. This problem, however, was already extensively discussed in Papers I and II, and the main arguments of this discussion will not be repeated here.

\section{Conclusions}

From our studies of the generation of transverse tube waves propagating along a single magnetic flux tube embedded in atmospheres of late-type stars with different metallicities, the following conclusions can be drawn.

1. The shapes of the computed wave energy spectra are similar for stars of the same effective temperature and gravity but different metallicity.

2. The effects caused by the mixing-length parameter and the tube magnetic field on the rate of generation of transverse tube wave are independent of stellar metallicity.

3. The effects of metallicity are important for stars with $\log g=5$ and $T_{\text {eff }}<6000 \mathrm{~K}, \log g=4$ and $T_{\text {eff }}<5500 \mathrm{~K}$, and $\log g=3$ and $T_{\text {eff }}<5000 \mathrm{~K}$, and the amount of the generated wave energy decreases roughly by an order of magnitude for every decrease of the metallicity by an order of magnitude.

4. For hot stars with $T_{\text {eff }}>6000 \mathrm{~K}$, the generated wave energy fluxes are practically independent of metallicity. However, for stars with $\log g=5$ and $T_{\text {eff }}>9500 \mathrm{~K}$, $\log g=4$ and $T_{\text {eff }}>8500 \mathrm{~K}$, and $\log g=3$ and $T_{\text {eff }}>$ $7500 \mathrm{~K}$, the wave generation becomes very sensitive to small changes in $T_{\text {eff }}$. As the convective velocities approach the sound speed in these stars the computed transverse wave fluxes are not very reliable.

5. The maximum wave energy flux generated in population II stars is about $3 \times 10^{9} \mathrm{erg} \mathrm{cm}^{-2} \mathrm{~s}^{-1}$ and it is practically the same for stars of different gravities and metallicities. 
6. Comparing our results to those obtained by Ulmschneider et al. (1999) for acoustic waves and by Musielak et al. (2001) for longitudinal tube waves shows that the effects of metallicity on the wave generation process are very similar for non-magnetic and magnetic waves.

Acknowledgements. This work was supported by NSF under grant ATM-0087184 (Z.E.M. and P.U.), by the DFG grant U157/25-3, and by NATO under grant CRG-910058 (P.U. and Z.E.M.). Z.E.M. also acknowledges the support of this work by the Alexander von Humboldt Foundation.

\section{References}

Alexander, D. R. 1975, ApJS, 29, 363

Alexander, D. R. 1989, ApJ, 345, 1014

Bohn, H. U. 1984, A\&A, 136, 338

Cox, A. N., \& Tabor, J. E. 1976, ApJS, 31, 271

Cuntz, M., Rammacher, W., Ulmschneider, P., Musielak, Z. E., \& Saar, S. H. 1999, ApJ, 522, 1053

Fawzy, D. E. 2001, Ph.D. Thesis, Univ. Heidelberg, Germany

Kurucz, R. L. 1992, in IAU Symp. 149, The stellar population of galaxies, ed. B. Barbuy, \& A. Renzini (Kluwer, Dordrecht)

Kurucz, R. L. 1996, in Workshop on model atmospheres and spectrum synthesis, ed. S. J. Adelman, F. Kupka, \& W. Weiss, ASP Conf. Ser., 108
Meyer-Hofmeister, E. 1982, Stellar Structure and Evolution, in Landolt-Börnstein, Neue Serie VI, Vol. 2b, Sect. 4.4.2.3.1., ed. K. Schaifers, \& H. H. Voigt (Springer, Berlin)

Musielak, Z. E., Rosner, R., \& Ulmschneider, P. 2001, ApJ, submitted

Musielak, Z. E., \& Ulmschneider, P. 2001, A\&A, 370, 541 (Paper I)

Musielak, Z. E., \& Ulmschneider, P. 2002, A\&A, 386, 606 (Paper II)

Rüedi, I., Solanki, S. K., Mathys, G., \& Saar, S. H. 1997, A\&A, 318,429

Saar, S. H. 1996, in IAU Symp. 176, Stellar Surface Structure, ed. K. Strassmeier, \& J. L. Linsky (Kluwer), 237

Solanki, S. K. 1993, Space Sci. Rev., 63, 1

Theurer, J. 1993, Thesis, Univ. Heidelberg, Germany

Ulmschneider, P., Fawzy, D. E., Musielak, Z. E., \& Stȩpień, K. 2001a, ApJ, 559, L167

Ulmschneider, P., Musielak, Z. E., \& Fawzy, D. E. 2001b, A\&A, 374,662

Ulmschneider, P., Theurer, J., \& Musielak, Z. E. 1996, A\&A, 315,212

Ulmschneider, P., Theurer, J., Musielak, Z. E., \& Kurucz, R. 1999, A\&A, 347, 243

Weiss, A. 1990, At. Data Nucl. Data Tables, 45(2), 209

Yorke, H. W. 1979, A\&A, 80, 308

Yorke, H. W. 1980, A\&A, 85, 215 